\title{
Optimization of Sintering Temperature for Maximizing Dimensionless Figure of Merit of La-Doped Strontium Titanate Thermoelectric Material in the Combination of Combustion Synthesis with Post Spark Plasma Sintering
}

\author{
Asami Kikuchi ${ }^{1, *}$, Lihua Zhang ${ }^{2}$, Noriyuki Okinaka ${ }^{3}$, Tsuyoshi Tosho ${ }^{4}$ and Tomohiro Akiyama ${ }^{3}$ \\ ${ }^{1}$ Graduate School of Engineering, Hokkaido University, Sapporo 060-8628, Japan \\ ${ }^{2}$ University of Science and Technology Beijing, Beijing 100083, P. R. China \\ ${ }^{3}$ Center for Advanced Research of Energy Conversion Materials, Hokkaido University, Sapporo 060-8628, Japan \\ ${ }^{4}$ Thermoelectric Device Development Inc., Noboribetsu 059-0003, Japan
}

\begin{abstract}
This paper describes thermoelectric properties of La-doped $\mathrm{SrTiO}_{3}$ prepared by using a combination of combustion synthesis (CS) with post spark plasma sintering (SPS), on which effects of sintering temperature were mainly examined. In experimental, combustion-synthesized (CSed) samples $\left(\mathrm{Sr}_{1-x} \mathrm{La}_{x} \mathrm{TiO}_{3}, x=0.08\right)$ were spark-plasma-sintered (SPSed) at temperatures from 1513 to $1663 \mathrm{~K}$ and the thermoelectric properties of sintered $\mathrm{Sr}_{0.92} \mathrm{La}_{0.08} \mathrm{TiO}_{3}$ were measured from room temperature to $1073 \mathrm{~K}$. In experimental sintering temperature range, sintering temperature didn't heavily affect the average grain size of sintered SLTO in the case that sintering temperature ranges from 1543 to $1603 \mathrm{~K}$. However, when sintering temperature rose over $1603 \mathrm{~K}$, the average grain size of sintered SLTO was dramatically affected and it increased with sintering temperature. With increasing sintering temperature, the effects of phonon scattering at grain boundaries decreased therefore, thermal conductivity of all sintered samples increased with sintering temperature. With increasing sintering temperature, electric conductivity of sintered SLTO increased and absolute value of Seebeck coefficient of sintered SLTO decreased because sintering at higher temperature caused the more oxygen defects which generate the larger carrier density. In conclusion, the product sintered at $1573 \mathrm{~K}$ showed the maximum of the dimensionless figure of merit of 0.16 at $1005 \mathrm{~K}$. [doi:10.2320/matertrans.M2010102]
\end{abstract}

(Received March 19, 2010; Accepted July 21, 2010; Published September 8, 2010)

Keywords: thermoelectric materials, perovskite oxide, combustion synthesis, spark plasma sintering

\section{Introduction}

The last decade has seen a big surge in research on thermoelectric oxide materials, with primary focus on improving the thermoelectric properties: electrical conductivity, $\sigma\left[\mathrm{Scm}^{-1}\right]$, Seebeck coefficient, $\alpha\left[\mu \mathrm{VK}^{-1}\right]$, and thermal conductivity, $\kappa\left[\mathrm{Wm}^{-1} \mathrm{~K}^{-1}\right] . \mathrm{SrTiO}_{3},{ }^{1-4)}$ a typical transition-metal perovskite oxide, is one of the most important ceramics and many researchers have studied thermoelectric properties of $\mathrm{SrTiO}_{3}$-based materials. ${ }^{5-9)}$

There have been many investigations on fabrication of $\mathrm{SrTiO}_{3}$ ceramics: wet-chemical method, ${ }^{10)}$ solid-state reaction (SSR) method and sol-gel method, which have already been widely used in the production of many materials. However, these conventional methods involve many processes therefore they are time and energy consuming. Zhang et al. ${ }^{1,11)}$ have proposed a combination of combustion synthesis $(\mathrm{CS})^{12)}$ and spark plasma sintering (SPS) ${ }^{13-18)}$ for the synthesis of thermoelectric materials. CS is one of chemical reaction method using the energy of exothermic reaction from raw materials. The principle of the CS is that once one end of the starting mixture is ignited, the exothermic sustainable reaction initiates and causes a combustion wave to propagate through the sample, and the desired product is finally formed without additional energy in pre-calcining step. In addition to energy saving, the CS can produce a non-equilibrium material when strong exothermic reaction makes rapid heating with quenching of the product. The obtained product is homogeneous with high purity. ${ }^{12)}$

As compared to conventional techniques such as hotpressed sintering, SPS ensures a very rapid heating rate

*Graduate Student, Hokkaido University and mass transfer speed, and allows the samples to be fabricated in a short period time at a relatively lower temperature. Consequently, SPS is the most suitable for fabrication of thermoelectric materials. ${ }^{18)}$ Sintering conditions, particularly sintering temperature affect its microstructures and properties seriously. ${ }^{19-21)} \mathrm{X}$. Dong et $a .^{20)}$ reported that the effect of sintering temperature on crystal structure and electrochemical performance of synthesized $\mathrm{La}_{0.80} \mathrm{Mg}_{0.20} \mathrm{Ni}_{3.75}$ alloy, and found that the density of the samples increases with increasing the sintering temperature. However, the effects of sintering temperature on the thermoelectric properties have never been researched thus far. To build up the fabricating method using CS with post SPS for thermoelectric materials, it is essential to survey the effects of sintering temperature on thermoelectric properties. Therefore, in this study, we investigated the effects of sintering temperature on the thermoelectric properties and microstructures of polycrystalline La-doped $\mathrm{SrTiO}_{3}$.

\section{Experimental Procedure}

Polycrystalline samples of La-doped $\mathrm{SrTiO}_{3}$ (SLTO) were prepared from $\mathrm{SrCO}_{3}(99.9 \%$ purity, Kanto Chemical, Tokyo, Japan), $\mathrm{TiO}_{2}$ (99.9\% purity, Kanto Chemical, Sakado, Japan), Ti (99.9\% purity, Kojundo Chemical, Sakado, Japan), $\mathrm{NaClO}_{4}$ (98.0\% purity, Sigma-Aldrich, St. Louis, US10), and $\mathrm{La}_{2} \mathrm{O}_{3}$ (99.9\% purity, Kojundo Chemical, Sakado, Japan). ${ }^{22)}$ After combustion-synthesis, the obtained products were pulverized into powders in a planetary ball mill (Pulverisette 6, Fritsch, Idor-Oberstein, Germany) operated at $350 \mathrm{rpm}$ for $40 \mathrm{~min}$ in air. The particle size of the obtained powders was within $5 \mu \mathrm{m}$. 
The obtained powders were filled in a cylindrical graphite die (inner diameter $=15 \mathrm{~mm}$ ) and pressed by using a graphite punch at $37.45 \mathrm{MPa}{ }^{22)}$ Then, pulverized powders were sintered by SPS (SPS-511S, Sumitomo Coal Mining, Tokyo, Japan) at a heating rate of $30 \mathrm{Kmin}^{-1}$ and a holding time of $15 \mathrm{~min}$ in vacuum. During sintering, the mechanical pressure was maintained at $34 \mathrm{MPa}$ by using plungers. In this study, sintering was carried out at different holding temperatures. The oxygen defect content $\delta$ in the sintered samples was determined by measuring the weight of the samples before and after heating in a furnace at $1373 \mathrm{~K}$ for $12 \mathrm{~h}$ in air. Heating was repeated twice. $\delta$ was calculated assuming that the samples were oxidized to $\mathrm{Sr}_{0.92} \mathrm{La}_{0.08} \mathrm{TiO}_{3.00}$ in furnace. The phase composition and morphology of the products were analyzed by using an X-ray diffractometer (Miniflex, Rigaku, Tokyo, Japan) and a scanning electron microscope (SEM) (JSM-7000F, JEOL, Tokyo, Japan). Lattice parameter of synthesized powders before SPS was calculated from X-ray diffraction (XRD) data obtained from another X-ray diffractometer (X'Pert Pro MPD, Royal Philips Electronics,
Eindhoven, Netherlands). $\sigma$ and $\alpha$ were simultaneously measured by using a Seebeck coefficient/electric resistance measuring system (ZEM-3, ULVAC-RIKO, Yokohama, Japan) from room temperature to $1073 \mathrm{~K}$ in $\mathrm{He}$ atmosphere. $\kappa$ was calculated as

$$
\kappa=D C_{\mathrm{p}} d
$$

where $D, C_{\mathrm{p}}$ and $d$ are the thermal diffusivity, heat capacity and experimental density, respectively. The densities of the samples were measured by Archimedes method, and the thermal diffusivity and heat capacity were measured by the laser flash thermal constant analyzer (TC-7000, ULVACRIKO, Yokohama, Japan) from room temperature to $1105 \mathrm{~K}$ in vacuum.

\section{Results and Discussion}

\subsection{Reaction analyses and crystal structure of combus- tion-synthesized and spark-plasma-sintered SLTO}

The equation of the CS reaction is given as follows: ${ }^{22)}$

$$
\begin{aligned}
& (1-x) \mathrm{SrCO}_{3}+(1-a) \mathrm{Ti}+a \mathrm{TiO}_{2}+(x / 2) \mathrm{La}_{2} \mathrm{O}_{3}+(4-4 a-x) / 8 \mathrm{NaClO}_{4} \\
& \quad \rightarrow \mathrm{Sr}_{1-x} \mathrm{La}_{x} \mathrm{TiO}_{3}+(1-x) \mathrm{CO}_{2}+(4-4 a-x) / 8 \mathrm{NaCl}
\end{aligned}
$$

In eq. (2), $x$ denotes the La-doping content and $a$ denotes the $\mathrm{TiO}_{2}$ content. In our study, $x$ and $a$ are equal to $0.08^{1)}$ and $0.25,{ }^{12)}$ respectively. The average particle size of the ground powders before SPS is found to be less than $5 \mu \mathrm{m}$ from the SEM image. ${ }^{22)}$ The average grain size of SLTO sintered at $1513,1543,1573,1603,1633$, and $1663 \mathrm{~K}$ was approximately $1.32,4.31,3.61,2.32,6.63$, and $23.5 \mu \mathrm{m}$, respectively. ${ }^{22)}$ The average grain size of sintered SLTO decreased above $1573 \mathrm{~K}$, but it increased again above $1603 \mathrm{~K}$. In experimental sintering temperature range, sintering temperature didn't heavily affect the average grain size of sintered SLTO in the case of sintering temperature ranges from 1543 to $1603 \mathrm{~K}$. However, when sintering temperature rose over $1603 \mathrm{~K}$, the average grain size of sintered SLTO was dramatically affected and it increased with sintering temperature.

Table 1 lists the lattice parameters of synthesized powders before SPS calculated from XRD data and comparison of sintering temperatures and bulk density after SPS. The value of $\delta$ for samples sintered at various sintering temperatures is

Table 1 Lattice parameters of synthesized powders before SPS calculated from X-ray diffraction (XRD) data and comparison of sintering temperatures and bulk density after SPS. The value of $\delta$ for samples sintered at various sintering temperatures is also shown in this table.

\begin{tabular}{ccccc}
\hline $\begin{array}{c}\text { Holding } \\
\text { temperature }[\mathrm{K}]\end{array}$ & $\begin{array}{c}\text { Lattice parameter } \\
\text { before SPS }[\mathrm{nm}]\end{array}$ & \multicolumn{2}{c}{$\begin{array}{c}\text { Bulk density after SPS } \\
\left(\mathrm{g} / \mathrm{cm}^{-3}\right)\end{array}$} & $\begin{array}{c}\text { The value of } \\
(\% \text { T.D. })\end{array}$ \\
\hline 1513 & & 5.03 & 96.27 & 0.0097 \\
1543 & & 5.15 & 98.57 & 0.034 \\
1573 & 0.3903 & 5.14 & 98.49 & 0.030 \\
1603 & & 5.09 & 97.56 & 0.026 \\
1633 & & 5.16 & 98.91 & 0.053 \\
1663 & & 5.09 & 97.55 & - \\
\hline
\end{tabular}

T.D. $=5.24 \mathrm{gcm}^{-3}$

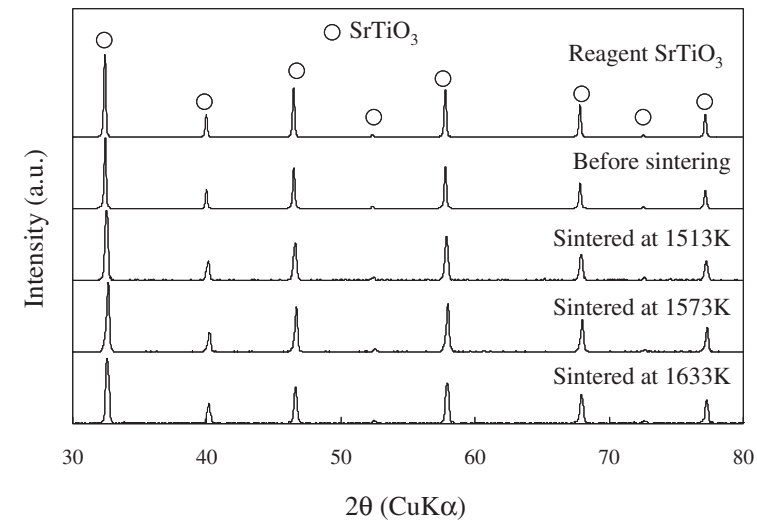

Fig. 1 X-ray diffraction patterns of SLTO before and after sintering at different temperatures, together with the data of reagent $\mathrm{SrTiO}_{3}$ for comparison.

also shown in this table. As shown in Table 1, density of all samples reached more than $96.27 \%$ of the theoretical density (T.D.) (T.D. $=5.24 \mathrm{gcm}^{-3}$ ). Lattice parameter of CSed SLTO powders was $0.3903 \mathrm{~nm}$. In this study, the samples were sintered at different temperatures from 1513 to $1663 \mathrm{~K}$. However, bulk SLTO was not formed at a sintering temperature of $1663 \mathrm{~K}$, because of the formation of many intercrystalline cracks. Thus, we concluded that sintering of SLTO was successfully carried out at temperatures from 1513 to $1633 \mathrm{~K}$.

Figure 1 shows XRD patterns of SLTO before and after sintering at different temperatures, together with the data of reagent $\mathrm{SrTiO}_{3}$ (99.9\% purity, Kojundo Chemical, Sakado, Japan) for comparison. All peaks in this figure very well correspond to that of reagent $\mathrm{SrTiO}_{3}$. This result indicates that SLTO with high purity was prepared by CS. There is no difference between the XRD patterns obtained before and 


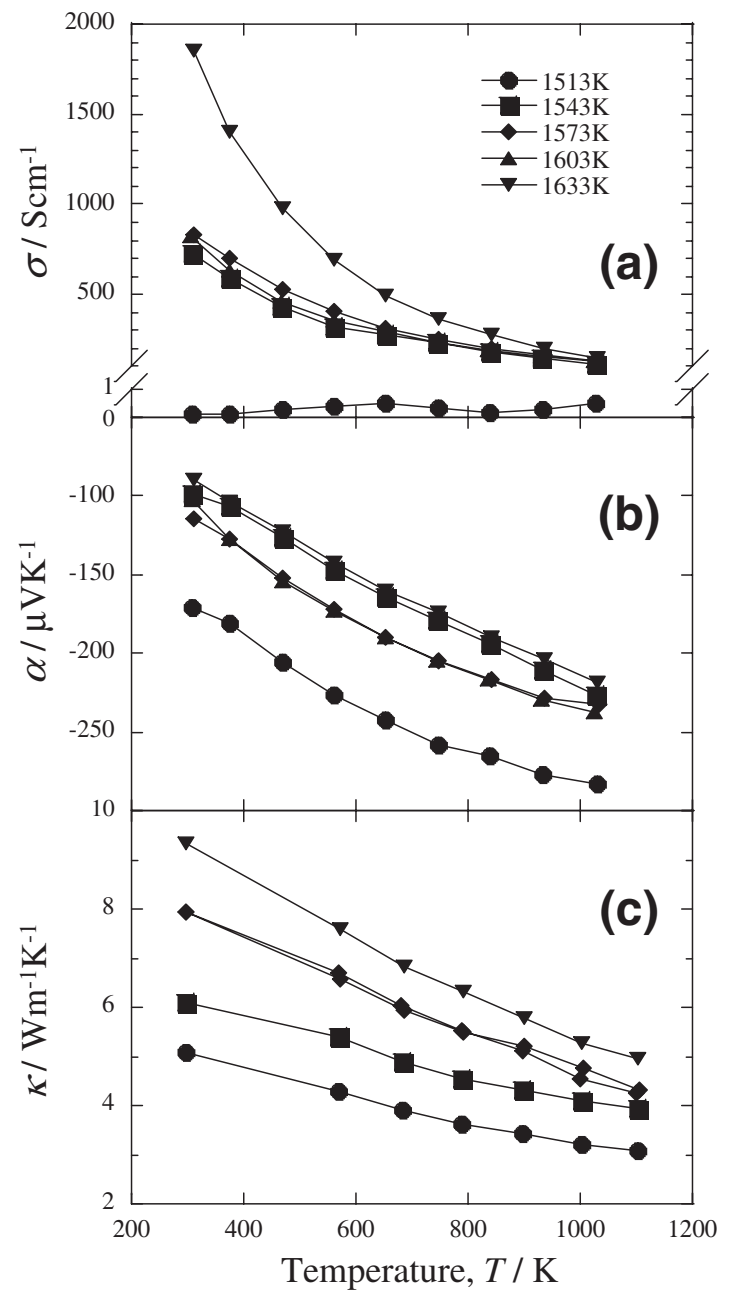

Fig. 2 Temperature dependence on (a) electric conductivity, ${ }^{22)}$ (b) Seebeck coefficient $^{22}$ and (c) thermal conductivity with various sintering temperatures.

after SPS, indicating that secondary-phase has not appeared during sintering. Also, we confirmed that the SEM image ${ }^{22)}$ didn't show secondary-phase in SPSed products.

\subsection{Temperature dependence of thermoelectric proper- ties of CSed and SPSed SLTO with various sintering temperatures}

Figures 2(a), (b) and (c) show the temperature dependence of $\sigma, \alpha$ and $\kappa$ of CSed and SPSed SLTO for various sintering temperatures, respectively. As temperature increased, $\sigma$ decreased and the absolute value of $\alpha$ increased, indicating a metallic behavior. In the experimental temperature range, with an increase in the sintering temperature, $\sigma$ increased and the absolute value of $\alpha$ decreased due to an increase in the carrier density. When a sample was sintered at high temperature in abutting contact with carbon in vacuum, a lot of oxygen inside particles were liberated. As a result, the sample changed into the nonstoichiometric compound of $\mathrm{Sr}_{0.92} \mathrm{La}_{0.08} \mathrm{TiO}_{3-\delta}$, where $\delta$ is the oxygen defect content. Oxygen defects generate electrons as carriers. As shown in Table 1, the largest value of $\delta$ is 0.053 in a sample sintered at $1633 \mathrm{~K}$, and the smallest value of $\delta$ is 0.0097 in a sample sintered at $1513 \mathrm{~K}$. The value of $\delta$ of the samples sintered at different temperatures also shows a similar tendency with

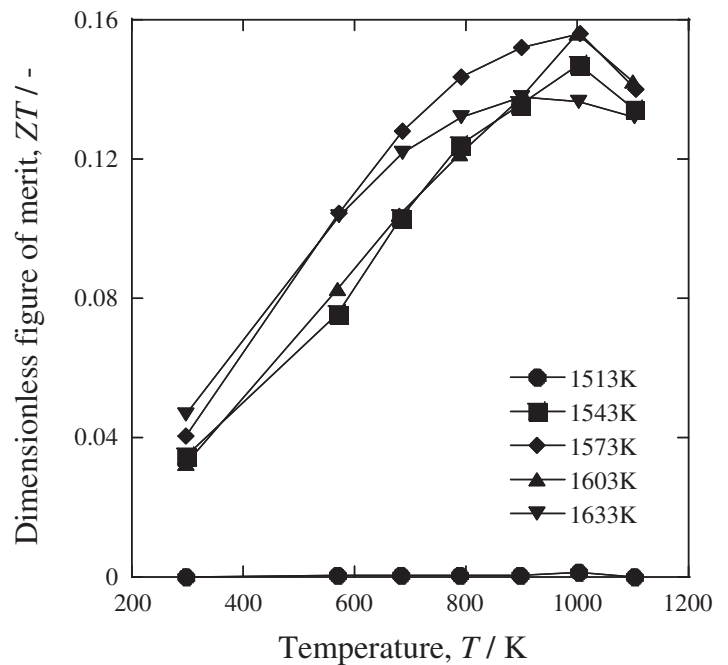

Fig. 3 Temperature dependence on dimensionless figure of merit with various sintering temperatures.

electric conductivity of the samples. As shown in Fig. 2(a), electric conductivity of samples sintered at 1543, 1573, and $1603 \mathrm{~K}$ were almost equal values. Therefore, the $\delta$ values were also almost equal. Thus, an increase in the sintering temperature leads to an increase in the oxygen defect content, thereby resulting in high $\sigma$. Thus, $\sigma$ increased and the absolute value of $\alpha$ decreased with an increase in the sintering temperature. Thermal conductivity of CSed and SPSed SLTO increased with sintering temperature. With an increase in sintering temperature, average grain size of CSed and SPSed SLTO increased, which caused the decrease of the effects of phonon scattering at grain boundaries. Therefore, thermal conductivity of CSed and SPSed SLTO increased with an increase in sintering temperature.

Figure 3 shows temperature dependence on dimensionless figure of merit with various sintering temperatures. In the temperature range considered, ZT of samples sintered at more than $1543 \mathrm{~K}$ showed peaks at around $1000 \mathrm{~K}$. ZT of sample sintered at $1513 \mathrm{~K}$ showed very low values due to its low electric conductivity shown in Fig. 2(c). Among the CSed and SPSed SLTO, sample sintered at $1573 \mathrm{~K}$ showed the maximum $\mathrm{ZT}$ of 0.16 at $1005 \mathrm{~K}$. These results indicate that for production of SLTO by the combination of CS and SPS, sintering should be done at $1573 \mathrm{~K}$.

\section{Conclusions}

We successfully synthesized La-doped $\mathrm{SrTiO}_{3}$ by the combination of CS with post SPS and figured out the effects of sintering temperature on the thermoelectric properties of polycrystalline $\mathrm{Sr}_{0.92} \mathrm{La}_{0.08} \mathrm{TiO}_{3}$ and following results were obtained:

(1) Spark-plasma-sintering of $\mathrm{Sr}_{0.92} \mathrm{La}_{0.08} \mathrm{TiO}_{3}$ was successfully carried out at 1513 to $1633 \mathrm{~K}$ in temperature. $\mathrm{Sr}_{0.92} \mathrm{La}_{0.08} \mathrm{TiO}_{3}$ powders were not sintered well at $1663 \mathrm{~K}$ due to the formation of many intercrystalline cracks.

(2) High-temperature sintering resulted in introduction of a lot of oxygen defects which produced electrons as carriers. Thus, samples sintered at higher temperature showed large 
electric conductivity. However, high-temperature sintering also caused an increase in thermal conductivity because of the increase in average grain size, therefore the most optimum sintering temperature maximized ZT was $1573 \mathrm{~K}$.

(3) With increasing temperature, ZT showed an increasing tendency and showed peaks at around $1000 \mathrm{~K}$. The maximum $\mathrm{ZT}$ of 0.16 at $1005 \mathrm{~K}$ was obtained from the sample sintered at $1573 \mathrm{~K}$.

\section{REFERENCES}

1) L. Zhang, T. Tosyo, N. Okinaka and T. Akiyama: Mater. Trans. 48 (2007) 1079-1083.

2) H. Muta, K. Kurosaki and S. Yamanaka: J. Alloy. Compd. 368 (2004) 22.

3) T. Maekawa, K. Kurosaki, H. Muta, M. Uno and S. Yamanaka: J. Alloy. Compd. 387 (2005) 56.

4) H. Muta, K. Kurosaki and K. Yamanaka: J. Alloy. Compd. 392 (2005) 306.

5) T. Okuda, K. Nakanishi, S. Miyasaka and Y. Tokura: Phys. Rev. B. Matter. 63 (2001) 113104-1.

6) H. Muta, K. Kurosaki and S. Yamanaka: J. Alloy. Compd. 350 (2003) 292.

7) H. Obara, A. Yamamoto, C. H. Lee, K. Kobayashi, A. Matsumoto and R. Funahashi: Jpn. J. Appl. Phys. 43 (2004) 540.
8) S. Ohta, T. Nomura, H. Ohta and K. Koumoto: J. Appl. Phys. 97 (2005) 034106-1.

9) S. Ohta, T. Nomura, H. Ohta, M. Hirano, H. Hosono and K. Koumoto: J. Appl. Phys. Lett. 87 (2005) 092108-1.

10) P. Balaya, M. Ahrens, M. L. Kienle, J. Maier, B. Rahmati, S. B. Lee and W. Sigle: J. Am. Ceram. Soc. 89 (2006) 2804.

11) L. Zhang, T. Tosho, N. Okinaka and T. Akiyama: Mater. Trans. 48 (2007) 2088-2093.

12) H. Ishikawa, K. Oohira, T. Nakajima and T. Akiyama: J. Alloy. Compd. 454 (2008) 384.

13) H. Simizu, M. Yoshinaka and K. Hirota: Mater. Res. Bull. 37 (2002) 1557.

14) T. Nishimura, M. Mitomo, H. Hirotsuru and M. Kawahara: J. Mater. Sci. Lett. 14 (1995) 1046.

15) I. J. Shon, Z. A. Munir, K. Yamazaki and K. Shoda: J. Am. Ceram. Soc. 79 (1996) 1875.

16) J. J. Petrociv and R. E. Honnell: J. Mater. Sci. 25 (1990) 4453.

17) Y. Liu, Y. Lin, Z. Shi, C.-W. Nan and Z. Shen: J. Am. Ceram. Soc. 88 (2005) 1337.

18) Y.-h. Lin, Z. Shi, C.-W. Nan, Y. Liu and J. Li: J. Am. Ceram. Soc. 90 (2007) 132.

19) Q. Hu, P. Luo and Y. Yan: J. Alloy. Compd. 459 (2008) 163.

20) X. Dong, F. Lti, L. Yang, Y. Zhang and X. Wang: Mater. Chem. Phys. 112 (2008) 596.

21) R.-C. Chang, S.-Y. Chu, Y.-P. Wong, C.-S. Hong and H.-H. Huang: J. Alloy. Compd. 456 (2008) 308.

22) A. Kikuchi, L. Zhang, N. Okinaka, T. Tosho and T. Akiyama: Mater. Trans. 50 (2009) 2675-2679. 